\title{
CARDIAC AND STRESS ASSESSMENT USING SVM CLASSIFIER
}

\author{
K.R.INDIRA \\ Assistant Professor \\ Computer Science and Engineering \\ P.S.R.R. Engineering College \\ Sivakasi, Tamilnadu, India.
}

\begin{abstract}
Electrocardiogram (ECG) is an electrical recording of the heart and used to measure the rate and regularity of the heartbeats. The obtained ECG signals are noisy, due to the loss of signal values, problems in the electrodes and natural addition of noises in the image. Initial filtering of the noise is done using wavelet transform. Features extracted are Mean, Median Moment, Skewness, Standard Deviation, Variance, Frequency, Entropy, Mode, Maximum, Minimum, Phase, Magnitude and Kurtosis. The Rpeaks are detected from the signals and the values are combined along with the previously calculated features. Optimizations are performed with the extracted feature that reduces the number of feature values by selecting the best feature values from the extracted feature values. Then classification is done using Support Vector Machine classifier. The classifier analyzes the feature values and identifies whether the input signal is normal signal or abnormal signal.
\end{abstract}

\begin{abstract}
Keywords: Electrocardiogram (ECG), Particle Swarm Optimization (PSO), Support Vector Machine (SVM), Wavelet Transform (WT).
\end{abstract}

\section{INTRODUCTION}

The ECG arrhythmia recordings utilized in this are obtained from the MIT-BIH arrhythmia database. This paper selects 14 ECG beat types form MIT-BIH arrhythmia database in which half of the samples are selected as training set and other half as the testing set. The ECG beat types include in this paper are Normal beat, Premature ventricular Contraction (PVC), Paced beat (PACE), right bundle branch block beat (RBBB), left bundle branch block beat (LBBB), atrial premature contraction (APC), ventricular flutter wave (VLWAV), and ventricular escape beat (VESC).

ECG signal is considered as a non-stationary signal. The most efficient techniques for signal noise elimination for such a non-stationary signal processing is the wavelet transformed especially for signal. Thresholding is used in wavelet domain to smooth out or to remove some coefficient of wavelet transform subsignals of the measured signal. Efficient selection of wavelet denoising parameters, such as wavelet function, decomposition levels, threshold function (method) and threshold selection to the signal denoising. These parameters are selected for low noise elimination performance. A Particle Swarm Optimization Algorithm (PSO) is used to search the wavelet denoising parameters in order to obtain the optimal ECG signal filtration efficiency.

The ECG signals are analyzed based on their shape and duration of the waveforms obtained from the patients. The analyzing of ECG signal is difficult because of their varying amplitudes with respect to time. The abnormalities caused due to stress leads to many heart related problem. In existing system the ECG signal are analyzed for diagnosing the cardiac abnormalities. The system should be well efficient in identifying the influence of stress arrhythmia because the chronic stress leads to several heart abnormalities. The accuracy in identification of disease should be high.

\section{RELATED WORK}

Mehrdad Javadi et al. (2013) proposed a novel ECG arrhythmia classification method using the complementary features of mixture of experts (ME) and Negatively Correlated Learning (NCL). Negative Correlation Learning and Mixture of Experts methods utilize different error functions for simultaneous training of negatively correlated Neural Networks. ECG features can be extracted in time domain, in frequency domain or represented as statistical measures. The wavelet transformation method is used to extract the features from the ECG signals [1].

Roshan joy Martis et al. (2013) proposed methodology involves computation of Discrete Cosine Transform (DCT) coefficients from the segmented beats of ECG, which is used for principal component analysis for dimensionality reduction. The average sensitivity was highest than $98.69 \%$, specificity was obtained than $99 / 91 \%$, and the classification accuracy was obtained than $99.52 \%$. The system for an automated classification of normal $(\mathrm{N})$ and 4 classes of abnormal(S, V, F and Q) beats. QRS complex was detected using pan Tompkins algorithm on the denoised ECG signal. Principal Component Analysis (PCA) is the linear dimensionality reduction technique is used to reduce the dimensionality of DCT coefficients [2].

Samit Ari et al. (2013) proposed an automatic ECG signal enhancement technique to remove noise. Stock well transform(stransform) is used to represent the noisy ECG signal in time frequency domain. Then masking and filtering techniques is used to remove unwanted noise from time frequency domain. The proposed method provides a better signal to noise ratio (SNR) and lower root mean square error (RMSE). The performance of different ECG enhancement techniques at $1.25 \mathrm{db}$ input SNR level are compared using analysis of variance based on statistical evaluations technique [3].

Joachim Behar et al. (2013) provided an automated algorithm to assess electrocardiogram (ECG) quality for both normal and abnormal rhythms is presented for false arrhythmia alarm suppression of intensive care unit (ICU) monitor. Signal Quality Indices (SQIs) were derived from the ECG segments and used as the input to a support vector machine classifier with Gaussian kernel. Alarms are triggered when parameters are not within a defined range whose default setting varies from one monitor to another. ECG records from three databases. The databases denoted DB1, DB2, and DB3 respectively. DB1 was used in order to train an ECG quality assessment model. DB2 was used to test how the model performs on arrhythmia records. 


\section{International Journal of Scientific Research in Engineering and Management (IJSREM)}

DB3 is used how effectively ECG quality assessment can identify artifactual signal [4].

Manab Kumar Das et al. (2013) ECG signal consist of several ECG beats and each ECG beat contains $P$ wave, QRS wave and T wave. The features are extracted using s-transform (ST) and wavelet transform (WT) separately from ECG beat. The extracted features are classified using multilayer perceptron neural network (MLP-NN). The average sensitivity performance of the proposed feature extraction technique for $\mathrm{N}, \mathrm{S}, \mathrm{F}, \mathrm{V}$ and $\mathrm{Q}$ are $95.70 \%, 78.05 \%, 49.60 \%, 89.68 \%$, and $33.89 \%$. Two types of feature are extracted in ECG cardiac cycle: morphological features and temporal features [5].

G.Ranganathan et al. (2012) presents the evaluation of mental stress assessment using heart-rate variability (HRV). Mental stress is accompanied by dynamic changes in autonomic nervous system (ANS) activity.HRV analysis is a popular tool for assessing the activities of autonomic nervous system. The wavelet decomposition and reconstruction techniques are used for removing noise and to extract time frequency features. The spectral features are identified by neuro fuzzy training mental stress assessment using fuzzy clustering techniques [6].

Marcin D.Bugdol et al. (2014) novel multimodal, behavioral biometric system that combines ECG and sound signal. The feature extraction methods from the ECG signal which are currently used in biometrics can be divided into two main groups as, methods based on fiducial points and methods not based on the fiducial points. The features evaluation has been separately carried out for each biometrics. The dimensionality reduction methods used in order to extract only the relevant features. The feature has been optimized with the Principal Component analysis and fisher discriminate analysis, the linear support vector machine. [7].

Kehan Zeng et al. (2014) particle swarm optimization is proposed to satisfy real life noise from heart sound (HS) signals. Short Time Fourier Transform (STFT) 12 is used to decompose each HS cycle into time frequency fragments are called granules. Cuboid is added to each granule to identify that it is a constituent HS or noise. The similar granules of HS cycles are close to each other in time frequency energy space. If number of granules reaches the threshold the center granule is identified as HS constituent and retained otherwise deleted. PSO is employed to find the optimal values of length, width, and height of the Cuboid. The extracted granules are merged into noise quasi free HS signal. [8].

Dimitris Giakoumis et al. (2013) proposed a novel subject dependent biosignal features with a view towards increasing the effectiveness of automatic psychological stress detection.. The proposed features use filtering of input signals, on the basis of ,rest signature" calculated from each baseline recording. These signatures are biosignal transformation to express each individual's baseline deviation from signal templates. They provided accuracy at the level of $95 \%$, significantly improved to the respective result (86.05\%) taken from common SC and ECG features that have been typically used in the past [9]

El-Sayed A.El-dahshan (2011) proposed an effective hybrid scheme for the denoising of electrocardiogram (ECG) signals corrupted by non-stationary noises using genetic algorithm (GA) and wavelet transform (WT). The wavelet denoising is used in noise reduction of multi channel high resolution ECG signal. The efficiency performance is evaluated using percentage root mean square difference (PRD) and signal to noise ratio (SNR). GA is used to optimize the entire range set of wavelet denoising parameter to an efficient ECG signal filtration. The noise reduction of a signal depends on the optimum value of the level of decomposition [10].

Himanshu Gothwal et al. (2011) presents a method to analyze electrocardiogram (ECG) signal, extract the features, for the classification of heart beats according to different arrhythmias. Fast Fourier transforms are used to identify the peaks in the ECG signal and then Neural Network are applied to identify the diseases. Then deflections in the ECG signal Q, R, S are identified and through these deflections QRS complex is identified which is important feature in identifying arrhythmias. The accuracy has been obtained as $98.48 \%$. High accuracy of the system makes it highly reliable and efficient [11].

Lukas Smital et al. (2013) the reduction of broad-band myopotentials (EMG) in ECG signals using the wavelet wiener filtering with noise free signal estimation. The dyadic stationary wavelet transform (SWT) in the wiener filter in estimating the noise free signal. The proposed AWWF algorithm provides better filtering than other tested algorithm based on simple wavelet wiener filtering. The algorithm is adaptive in two ways, the first adaptive lies in the division of the signal into individual segments, each with an approximately constant level of noise. These parameters are the decomposition level, filter banks, thresholding method and threshold value. The second adaptation is within individual segments. It lies in adaptive setting of threshold value based on the standard deviation of the noise at decomposition level [12].

Suranai Poungponsri, et al. (2013) Electrocardiogram (ECG) signals have been widely used in clinical studies to detect heart diseases. Conventional methods for ECG noise removal do not yield satisfactory results due to the non-stationary nature of the associated noise sources and their spectral overlap with desired ECG signals. An adaptive filtering approach based on discrete wavelet transform and artificial neural network is proposed for ECG signal noise reduction. This new approach combines the multi- resolution property of wavelet decomposition and the adaptive learning ability of artificial neural networks, and fits well with ECG signal processing applications. [13].

\section{PROPOSED WORK}

Our proposed system is to identify the Cardiac and stress arrhythmia from ECG signal. The accuracy in identifying the cardiac and stress abnormalities should be high. For this purpose the wavelet transform is used to remove the noise from the ECG Signal. The wavelet coefficients are used for feature extraction. The features extracted were optimized by the PSO algorithm. The classifiers used are support vector machine classifiers are used for classifying the abnormality of the ECG signal. The results of the classifier are compared based on their performance measures.

a. Database

The ECG arrhythmia recordings utilized in this are obtained from the MIT-BIH arrhythmia database. This paper selects 14 ECG beat types form MIT-BIH arrhythmia database in which half of the samples are selected as training set and other half as the testing set. ECG signals were initially preprocessed using Daubechies Wavelet4. 


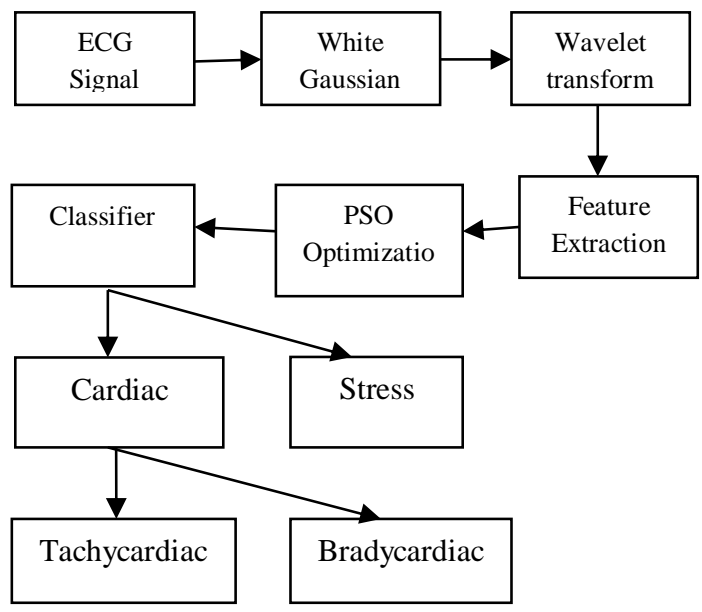

Figure 1. Architecture of Proposed Work

\section{b. Wavelet transform}

Preprocessing is the process of removing the noise present in the ECG signals. By means it increases the efficiency. The Daubechies wavelets are the orthogonal wavelets defining a discrete wavelet transform. The WT is used to analyze the signal by decomposing the signal into an approximation and detailed coefficients.

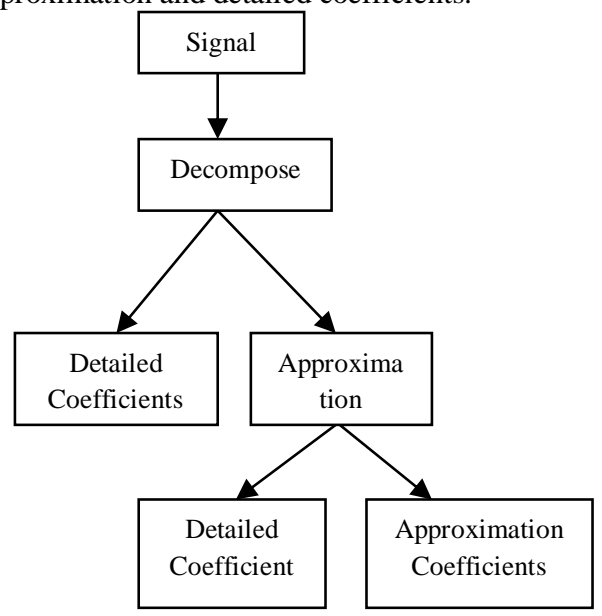

Figure 2. Wavelet Decomposition

$$
W_{m, k}(t)=\frac{1}{\sqrt{a}} W\left(\frac{1}{a}(t-b)\right)
$$

for discrete parameters of dilation $a=2 m$ and translation $b$ $=k 2 \mathrm{~m}$. Wavelet dilation, which is closely related to spectrum compression, enables local and global signal analysis.

Wavelet Transform

$$
\text { velocity }=w * \text { velocity }+c 1 *\left(r *\left(\text { local }_{\text {best }}^{\text {position }} \text { current }_{\text {position }}\right)\right)+c 2 *\left(r 2 *\left(\text { global }_{\text {best }_{\text {position }} \text { current }_{\text {position }}}\right)\right)
$$

The main advantages of particle swarm optimization is simplicity and easy implementation, the algorithms can be used widely in the fields such as function optimization. The basic particle swarm optimization algorithm consists of " $n$ " particles, and the position of each Particle stands for the potential solution in D-dimensional space. The particles change its condition according to the Following three principles: (1) to

c. $\quad$ Noise Removal using $d b 4$

The WT is used for wavelet decomposition of the ECG signal. The Daubechies ( $\mathrm{Db}$ ) wavelet has been selected for denoising the ECG signal because the wavelet has been similarity with QRS complex and the energy spectrum is concentrated around low frequencies. The signal is decomposed or four levels using Db4 wavelet. The white Gaussian noise is cancelled from the ECG signal for the purpose of efficient classification of disease. The positions of R peak of the ECG signal are marked.

\section{d. Feature Extraction}

The Feature selection has three steps in selecting the signal: Screening, Ranking, and Selecting. In screening, it removes unimportant and problematic predictors and records or cases, such as predictors with many missing values or predictors with too much or too little ranks to be useful. Ranking, Sorts remaining predictors and assigns by preserving only the most important predictors and filtering or excluding all others. The Features Extracted is Mean, Moment, Skewness, Standard Deviation, Variance, Kurtosis, Frequency, Maximum, Minimum, Phase, Mode, Entropy, Median, Energy, and Magnitude

\begin{tabular}{lcccccc}
\hline Sno & Mean & Median & Skewness & Kurtosis & Variance & Frequency \\
\hline ECG1 & $1.9280 \mathrm{e}+003$ & $1.9171 \mathrm{e}+003$ & 4.0312 & 23.4555 & $7.2914 \mathrm{e}+003$ & $3.3635 \mathrm{e}+009$ \\
ECG2 & $1.9739 \mathrm{e}+003$ & $1.9503 \mathrm{e}+003$ & 4.0815 & 24.8385 & $1.4371 \mathrm{e}+004$ & $3.5391 \mathrm{e}+009$ \\
ECG3 & $1.9612 \mathrm{e}+003$ & $1.9374 \mathrm{e}+003$ & 2.2226 & 9.7170 & $1.5634 \mathrm{e}+004$ & $3.4952 \mathrm{e}+009$ \\
ECG4 & $1.9772 \mathrm{e}+003$ & $1.9432 \mathrm{e}+003$ & 1.5921 & 7.5652 & $2.3487 \mathrm{e}+004$ & $3.5591 \mathrm{e}+009$ \\
ECG5 & $1.7095 \mathrm{e}+003$ & $1.7089 \mathrm{e}+003$ & 0.9575 & 5.9412 & $6.2145 \mathrm{e}+003$ & $2.6503 \mathrm{e}+009$ \\
\hline
\end{tabular}

Table 1. Feature values extracted from the ECG signal

e. $\quad$ Particle Swarm Optimization (PSO)

Particle Swarm Optimization is used to reduce the features for better optimization. 14 features are input for the optimization and the features are reduced optimized features for each input signal. The parameters are initialized based on global and local fitness. Then the random vectors component are assigned as $[0,1], \mathrm{r} 1$ and $\mathrm{r} 2$ are the random variables that allows stochastic search in the search space. The particles velocities and position are initialized by the current position. The particles initial position is evaluated by the local best position and global best position. The velocity of each particle is updated by the local best position, global best position and current position of the each 
keep its inertia (2) to change the condition according to its most optimist position (3) to change the condition according to the swarm's most optimist position.

\section{f. Support Vector Machine Classifier}

Support Vector Machine is a new method for the classification of both the linear and nonlinear data. SVM is an algorithm that, it uses a nonlinear mapping to transform the original training data into a higher dimension. It searches for the linear optimal separating hyperplane ("Decision Boundary", separating the tuples of one class from another). Nonlinear mapping to a sufficiently high dimension, data from two classes can always be separated by a hyperplane. The SVM finds this hyperplane using support vector and margins. In SVM when the dataset has exactly two classes. An SVM classifier data by finding the best hyperplane, that separates all data points of one class from those of the other class. The best hyperplane for an SVM means the one with the largest margin between the two classes. Margin means the maximal width of the slab parallel to the hyperplane that has no interior data points. Optimized feature sets are given as input to the SVM. The SVM classification is used to classify the cardiac arrhythmia. SVM is the machine learning techniques to classify the cardiac arrhythmia. SVM identifies the best separating hyperplane between the two classes of the training samples within the features space by focusing on the training cases placed at the edge of the class descriptors. In this, not only an optimal hyperplane is fitted, but also less training samples are effectively used; thus high classification accuracy is achieved with small training set.

The support vector are the data points that are closest to the separating hyperplane, + indicates the data points of type 1 and -indicates data points of type -1 .

\section{EXPERIMENTAL RESULTS}

The dataset were extracted from the MIT-BIH database. The dataset contains both the cardiac and stress arrhythmia database. The classification performance of the proposed ECG arrhythmia classification scheme was validating by the ECG beat types from the MIT-BIH arrhythmia database. Diseases considered for the experimentation are Tachycardia, Bradycardia, Ventricular Tachycardia, Ventricular Fibliration, etc, The ECG beats samples were randomly separated into two subsets: One half of the samples were selected as the training subset and the other half as the testing subset. To evaluate the performance of the proposed classification scheme, three common measures of sensitivity, specificity, and accuracy are used and defined as follows:

Sensitivity $(\%)=\mathrm{TP} / \mathrm{TP}+\mathrm{FN} * 100$

Specificity $(\%)=T N / T N+F P * 100$

Accuracy $=T P+T N / T P+T N+F P+F N * 100$

Where TP is True Positive, FN is False Negative, TN is True

Negative, and FP is False Positive.

Sensitivity includes the values of true positive rate, Specificity includes the values of true negative rate, and Accuracy includes the total number of predictions that were correct.

Sensitivity $(\%)=100$

Specificity $(\%)=95.2381$

Accuracy $(\%)=96.2963$

In this comparison chart that shows the performance of the proposed system by the (\%) values it has been performed with sensitivity, specificity and accuracy.

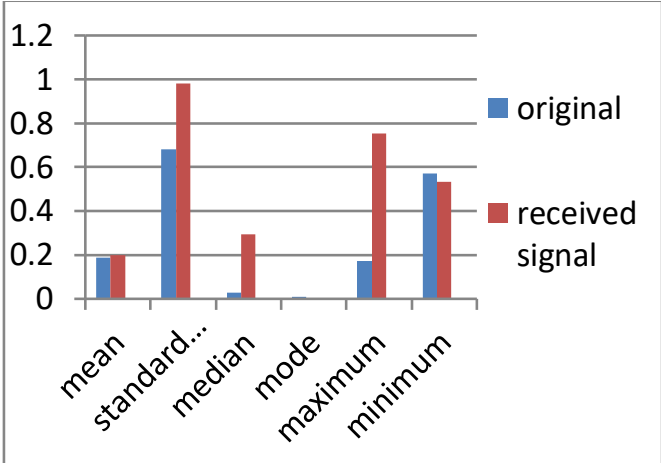

Figure 3 Comparison chart for the Feature Values

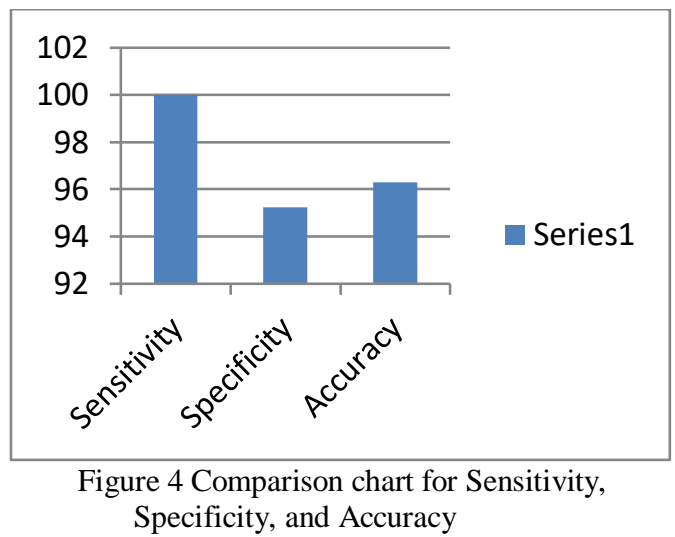

5. CONCLUSION

The denoising technique for ECG signal is proposed based on Particle Swarm Optimization and Wavelet transform. The input ECG signal is preprocessed to remove the noise from it. The Daubechies wavelet transform is used for removing the noises. The peaks in the ECG signal are detected for the purpose of extracting the features from the signal. The features are trained and Support Vector Machine is used for classification of the cardiac and stress arrhythmia.

\section{REFERENCES}

[1]. Mehrdad Javadia, Seyed Ali Asghar Abbaszadeh Aranib, Atena Sajedina, Reza Ebrahimpourb ,"Classification of ECG arrhythmia by a modular neural network based on Mixture of Experts and Negatively Correlated Learning “ Elsevier- Biomedical Signal Processing and Control 8 (2013).

[2]. Roshan Joy Martis, U. Rajendra Acharya, Choo Min Lim Jasjit S.Suri, " Characterization of ECG beats from cardiac rrhythmia using discrete cosine transform in PCA framework " Elsevier-KnowledgeBased System 45 (2013).

[3]. Samit Ari, ManabKumarDas, AnilChacko, "ECG signal enhancement using S-Transform", Elsevier-Computer in Biology and Medicine 43 (2013).

[4]. Joachim Behar, Julien Oster, Qiao Li, and Gari D. Clifford, Senior Member, IEEE ," ECG Signal Quality During Arrhythmia and Its Application to False Alarm Reduction “,IEEE

[5]. Manab Kumar Das_, Samit Ari, "ECG Beats Classification Using Mixture of Features ", IEEE transactions on biomedical engineering. 
[6]. G. Ranganathan, R. Rangarajan, V. Bindhu, "Estimation of heart rate signals for mental stress assessment using neuro fuzzy technique ", Elsevier- Applied Soft Computing 12 (2012).

[7]. Marcin D. Bugdol, Andrzej W. Mitas, "Multimodal biometric system combining ECG and sound signals ", Elsevier- Pattern Recognition 38 (2014).

[8]. Kehan Zeng, Mingchui Dong a, "A novel cuboid method with particle swarm optimization for real-life noise attenuation from heart sound signals “, Elsevier-Expert Systems with Application 41 (2014)

[9]. Dimitris Giakoumis, Dimitrios Tzovaras, George Hassapis, „Subjectdependent biosignal features for increased accuracy in psychological stress detection, ,

Science Direct-J-Human Computer Studies 71(2013).

[10]. Lukas Smital , Martin Vitek ,Jiri Kozumplik, and Ivo Provaznik, Member IEEE, „Adaptive Wavelet Wiener Filtering of ECG Signals", IEEE transaction on biomedical engineering, vol, 60, No.2,(2013). 